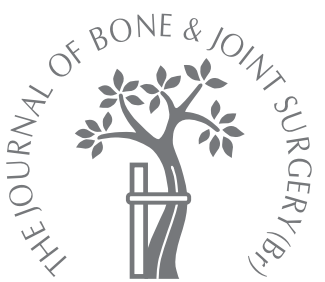

T. N. Board, P. Rooney, P. R. Kay

From Wrightington Hospital, Wigan, England

\title{
Strain imparted during impaction grafting may contribute to bony incorporation
}

\author{
AN IN VITRO STUDY OF THE RELEASE OF BMP-7 FROM \\ ALLOGRAFT
}

In order to investigate the osteoinductive properties of allograft used in impaction grafting and the effect of strain during impaction on these properties, we designed an in vitro experiment to measure strain-related release of bone morphogenetic protein-7 (BMP-7) from fresh-frozen femoral head allograft. A total of $4010 \mathrm{~mm}$ cubes of cancellous bone were cut from ten samples of fresh-frozen femoral head. The marrow was removed from the cubes and the baseline concentrations of BMP-7 were measured. Specimens from each femoral head were allocated to four groups and subjected to different compressive strains with a material testing machine, after which BMP-7 activity was reassessed. It was present in all groups. There was a linear increase of $102.1 \mathrm{pg} / \mathrm{g}$ (95\% confidence interval 68.6 to 135.6 ) BMP-7 for each $10 \%$ increase in strain. At $\mathbf{8 0} \%$ strain the mean concentration of BMP-7 released (830.3 pg/g bone) was approximately four times that released at $20 \%$ strain. Activity of BMP-7 in fresh-frozen allograft has not previously been demonstrated. This study shows that the freezing and storage of femoral heads allows some maintenance of biological activity, and that impaction grafting provides a source of osteoinductive bone for remodelling.

We have shown that BMP-7 is released from fresh-frozen femoral head cancellous bone in proportion to the strain applied to the bone. This suggests that the impaction process itself may contribute to the biological process of remodelling and bony incorporation.

T. N. Board, MRCS, MSc(OrthEng), FRCS(Orth), Consultant Orthopaedic Surgeon and Honorary Senior Lecturer at Manchester University

P. R. Kay, FRCS, Consultant Orthopaedic Surgeon and Senior Lecturer at Manchester University

The Centre for Hip Surgery Wrightington Hospital, Appley Bridge, Wigan WN6 9EP, UK.

- P. Rooney, BSc, PhD, Research and Development Manager, Honorary Lecturer at Manchester University Tissue Services, NHS Blood and Transplant, Estuary Banks, Liverpool L24 8RB, UK.

Correspondence should be sent to $\mathrm{Mr}$ T. N. Board; e-mail: tim@timboard.co.uk

(c)2008 British Editorial Society of Bone and Joint Surgery doi:10.1302/0301-620X.90B6 $20234 \$ 2.00$

$J$ Bone Joint Surg $[\mathrm{Br}]$ 2008;90-B:821-4. Received 19 September 2007; Accepted after revision 21 January 2008
Impaction grafting is a successful technique for restoring the lost bone stock in both the femur and the acetabulum during revision hip arthroplasty. ${ }^{1-4}$ Up to $100 \%$ survival of the implant has been reported at ten years using this technique. ${ }^{2}$ The procedure involves progressive compaction of morcellised cancellous bone chips into the femoral canal or acetabular cavity. The bone most commonly used to create the morcellised graft is fresh-frozen femoral head allograft. ${ }^{5}$ In order to achieve long-term survival of the prosthesis, two requirements must be met. First, initial stability must be achieved at the time of surgery, and second, the allograft must be incorporated into the host skeleton and undergo remodelling in response to the stress applied to it. If either of these steps fails, the implant will subside and further revision will be required. It has been shown in histological studies that impaction grafted bone does incorporate, but to a variable degree. ${ }^{6-9}$ We wished to investigate the potential contribution of the impaction process itself to the incorporation of allograft bone. It was hypothesised that, during impaction, the microfractures created in the bone chips may allow the exposure and release of factors in the non-collagenous matrix that would help to stimulate the subsequent incorporation and remodelling. We decided to investigate the release of bone morphogenetic protein-7 (BMP-7) or osteogenic protein-1 (OP-1), as this is a well-studied member of the transforming growth factor (TGF)- $\beta$ superfamily, and in recombinant form has been used to stimulate healing in spinal fusions, ${ }^{10}$ segmental defects of long bone ${ }^{11}$ and nonunions of the scaphoid. ${ }^{12}$ The use of additional recombinant BMP-7 in revision arthroplasty of the hip has also been studied. ${ }^{13-16}$

\section{Materials and Methods}

Preparation of the bone cubes. The bone used was taken from fresh-frozen femoral heads which had been cleared for use in research by the quality control team of Tissue Services at the National Blood Service, Liverpool, United Kingdom. The consent from donors included use for research and development.The heads were not suitable for clinical use, usually because of incomplete serological tests. Ten femoral heads were chosen at random and 


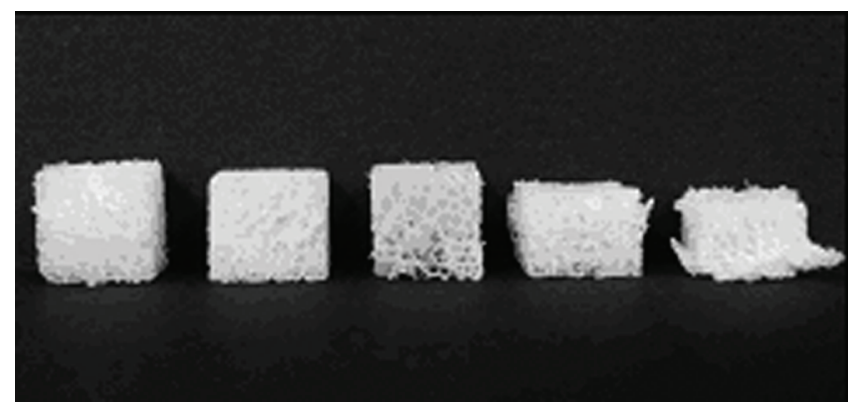

Fig. 1

Bone cubes following different levels of strain: from left to right $0 \%$, $20 \%, 40 \%, 60 \%$ and $80 \%$. All cubes showed a degree of recoil after strain, as demonstrated by the final resting heights.

Table I. Summary statistics of each strain group

\begin{tabular}{llll}
\hline & $\begin{array}{l}\text { Mean (SD) } \\
\text { baseline } \\
\text { (pg/g bone) }\end{array}$ & $\begin{array}{l}\text { Mean (SD) } \\
\text { after strain } \\
\text { (pg/g bone) }\end{array}$ & $\begin{array}{l}\text { Difference } \\
\text { (pg/g bone) }\end{array}$ \\
\hline $80 \%$ & $425.2(435.7)$ & $830.3(466.2)$ & $405.1(455.7)$ \\
$60 \%$ & $373.5(287.8)$ & $570.7(507.9)$ & $197.2(499.6)$ \\
$40 \%$ & $299.1(214.9)$ & $333.2(261.2)$ & $34.2(265.1)$ \\
$20 \%$ & $317.3(247.2)$ & $216.6(199.1)$ & $-100.7(102.8)$ \\
\hline
\end{tabular}

thawed at room temperature for two hours prior to dissection of the soft tissue. They were then cut into $10 \mathrm{~mm}$ cubes using a low-speed handsaw and a modified mitre block. The cubes were cut in line with the axis of the femoral neck. The orientation of each bone cube was noted, so that subsequent strain could be applied in the vertical direction. A total of 40 bone cubes were produced.

In order to remove the marrow content from the cubes they were first washed in $200 \mathrm{ml}$ water at room temperature. They were then placed individually in universal tubes and centrifuged at $1850 \mathrm{~g}$ for 15 minutes. They were then removed from the tubes and placed in $30 \mathrm{ml}$ distilled water at $37^{\circ} \mathrm{C}$ in clean universal tubes. These tubes were agitated at $200 \mathrm{rpm}$ in a desktop orbital incubator, maintaining a constant temperature of $37^{\circ} \mathrm{C}$, for one hour. The centrifuge step was repeated and the cubes were then weighed accurately, placed in clean universal tubes, and frozen at $-20^{\circ} \mathrm{C}$ until required. Throughout this washing procedure the tubes were labelled to maintain the identity of each cube.

Experimental method. On the morning of the experiment the cubes were allowed to thaw at room temperature for one hour. To each universal tube, $4 \mathrm{ml}$ of sterile distilled water was added $\left(\right.$ at $\left.37^{\circ} \mathrm{C}\right)$. The tubes were then agitated at $200 \mathrm{rpm}$ in the desktop incubator for two hours. The wash fluid was then removed, placed in labelled tubes and stored at $-20^{\circ} \mathrm{C}$ until required. These tubes were labelled 'wash 1 '. The cubes were then allocated to one of four groups, with one cube from each head in each group, giving ten cubes in each of the four groups. A universal testing machine (Lloyds Instruments, Fareham, United Kingdom) was then used to impart a predefined strain to the individual cubes. The testing machine was set up so that the base consisted of a flat metal block and the cross-head attachment was a similarly flat metal plate, parallel to the base-plate. All the cubes in a group received the same level of strain: either $80 \%, 60 \%, 40 \%$ or $20 \%$ (Fig. 1). The cubes were strained individually after accurate measurement of all dimensions using the vernier callipers. The universal testing machine was set to a preload of $9 \mathrm{~N}$ and a strain rate of $10 \mathrm{~mm} / \mathrm{min}$. Following the application of strain, the cubes were returned to clean universal tubes and a further $4 \mathrm{ml}$ of distilled water at $37^{\circ} \mathrm{C}$ was added. The tubes were agitated at $200 \mathrm{rpm}$ for a further two hours. The fluid was removed and stored in separate, universal containers. These were labelled 'wash 2' and stored at $-20^{\circ} \mathrm{C}$ until required.

BMP-7 assay. The concentration of BMP-7 in all wash samples was determined using a commercially-available enzyme-linked immunosorbent assay (ELISA) kit (RayBiotech Inc, Norcross, Georgia). All samples were run in duplicate. Where the BMP-7 levels were below the limit of detection by the ELISA $(10 \mathrm{pg} / \mathrm{ml})$ a standard value of $5 \mathrm{pg} / \mathrm{ml}$ was assigned to prevent negative values interfering with the statistical calculations.

Statistical analysis. Statistical analysis was performed using Excel (Microsoft Corporation, Redmond, Washington) and SPSS 14.0 (SPSS Inc., Chicago, Illinois). The differences between groups were assessed using mixed modelling. Fixed (strain group and baseline BMP-7 value) and random (head) effects were estimated using restricted maximum likelihood.

\section{Results}

BMP-7 was present in all samples of femoral heads tested. The mean baseline concentration of BMP-7 for all cubes was $353.8 \mathrm{pg} / \mathrm{g}$. The SD of 300.3 (11.8 to 1442.1 ) reflects the wide variation in concentrations between samples. The release of BMP-7 in relation to strain was calculated by subtracting the calculated release after strain from the baseline values. The mean, SD and the change in the level of BMP per strain group are shown in Table I. The mean and variability of the BMP-7 showed differences between the four groups.

There was a statistically significant effect of strain on change in BMP-7 ( $p<0.0001)$, in the form of a linear increase of $102.1 \mathrm{pg} / \mathrm{g}$ (95\% confidence interval (CI) 68.6 to 135.6 ) for each $10 \%$ increase in strain. There was no evidence of any non-linearity in the effect $(p=0.36)$. The between-head SD was estimated to be 289.7 , whereas the estimated within-head SD was 234.9.

\section{Discussion}

We have shown that BMP-7 is present in fresh-frozen femoral head allograft. Although BMPs have been shown to be 
present in demineralised bone matrix ${ }^{17}$ this has not previously been demonstrated in fresh-frozen femoral head allograft and is a reflection of the osteoinductive properties of this type of graft.

The results also indicate that strain or microfracture induces the release of BMP-7 from fresh-frozen femoral head allograft. This is a phenomenon that has not previously been described in the literature. We observed a significant increase in the release of BMP-7 with increasing strain $(\mathrm{p}<0.0001)$. This increase was $102.1 \mathrm{pg} / \mathrm{g}$ bone per $10 \%$ increase in strain.

Bone morphogenetic proteins are sequestered in the bone matrix during osteogenesis and are bound to various types of collagen, including type I, IV and IX. ${ }^{18}$ The proposed mechanism of release as observed in this study is that during strain, microfractures are created, exposing fresh areas of bone matrix. Each microfracture exposes some new collagen strands to the surrounding micro-environment. The BMPs present on the collagen are also exposed. The openpore nature of the cancellous bone used in these experiments allows fluid to flow around these newly-exposed surfaces, washing off any cytokines that have been exposed into either solution or suspension. This mechanism is passive and is as equally applicable to fresh bone as any other form of bone in which BMPs are present.

In impaction grafting the process of impaction produces a biomechanically stable bed by creating interdigitation between bone particles. The aim of this experiment was to define whether the impaction process contributes to the biological incorporation of the graft bone. Having shown that BMP-7 is released following strain and microfracture, we can now postulate that the process itself may be beneficial to incorporation of the bone. Typical dosing regimens for in vivo use of recombinant BMPs are of the order of milligrams, ${ }^{19}$ rather than the picogram level found in our study. However, it has been noted that the osteoinductive properties of recombinant BMPs are reduced compared with purified BMPs, ${ }^{20}$ and although this study only measured BMP-7, this is a reflection of all the other BMPs that will be present in the matrix. Indeed, a study into the use of human BMPs for nonunion in long bones indicated that $100 \mathrm{ng}$ of endogenous BMP effected union in 20 of 30 patients. $^{21,22}$ A femoral head weighing $80 \mathrm{~g}$ would yield approximately $66 \mathrm{ng}$ of BMP-7 after impaction, and combined with the other BMPs present, this dose may have a significant effect. In addition to the release of growth factors after impaction, the compacted graft will also act as a slow-release depot for BMPs. As the bone is resorbed and remodelled, so the matrix will be broken down, releasing growth factors.

In terms of the observed quantity of BMP-7 measured in the samples, it is currently not known how much BMP-7 is present within a given amount of bone. However, one study showed that $20 \mu \mathrm{g}$ of highly purified BMP could be produced from $10 \mathrm{~kg}$ of bovine bone by heparin-sepharose chromatography and reverse-phase high-pressure liquid chromato- graphy. ${ }^{23}$ The largest release of BMP-7 in our experiment was $830.3 \mathrm{pg} / \mathrm{g}$ bone after the application of $80 \%$ strain. If we take $20 \mu \mathrm{g}$ per $10 \mathrm{~kg}$ bone to be indicative of BMP content, this equates to $2 \mathrm{ng} / \mathrm{g}$ bone. The $830.3 \mathrm{pg} / \mathrm{g}$ would therefore represent $42 \%$ of the total BMP present in the bone, which seems unlikely. However, the technique used to quantify the BMP-7 in this study, namely ELISA, is highly sensitive and the guanidinium chloride extraction process used by Wang et $\mathrm{al}^{23}$ may underestimate the total amount of BMP present. Furthermore, recent studies of BMP in demineralised bone matrix have indicated levels of BMP-7 of $227 \mathrm{ng} / \mathrm{g} .{ }^{24}$ As the non-organic mineral content of bone is known to be $60 \%$ of the weight, extrapolation indicates that there should be 90 ng BMP-7 per gram of bone. The observation of $830.3 \mathrm{pg} / \mathrm{g}$ bone therefore only accounts for $0.92 \%$ of the total BMP-7, a figure that seems more realistic.

The bone used for this experiment was taken from freshfrozen femoral heads procured from live donors at the time of primary hip replacement. Although many of these donors are elderly, whose bone is not always of good quality in terms of bone mineral content, and the articulating surface itself is, by definition, osteoarthritic, these heads are most commonly used in impaction grafting, which is the focus of this research. It is therefore logical to use this bone in these cytokine release experiments.

We have shown that the change in BMP-7 is markedly variable between femoral heads, even having accounted for the baseline value and strain effects (SD 289.7). However, the variability between individual cubes of bone is of a similar magnitude (SD 234.9). There are a number of possible explanations for this effect. There may be a relationship between age and cytokine sequestration in the matrix. However, as all of the femoral heads were procured from patients undergoing primary total hip replacement, this group is from a relatively narrow age range, precluding this analysis. Alternatively, the presence of disease such as osteoarthritis may have a significant effect on cytokines in the matrix. Although all the femoral heads showed evidence of osteoarthritis, both the extent and the cause of degeneration were variable. The variability between individuals observed in all the tests, from the haemoglobin levels to the bone mass, is unlikely to be the sole explanation for the large variation observed, as the mean concentration of BMP-7 for the femoral heads varied by a factor of 10 . No information on this variation in cytokine sequestration between individuals, or in different disease states, is available in the literature, although two recent studies used similar ELISA techniques to show marked variability in different BMPs in preparations of demineralised bone matrix. ${ }^{17,24}$ Other confounding factors, such as differences in the treatments of the 40 bone cubes, were kept to a minimum by the simultaneous processing of all cubes in the marrow removal and incubation wash stages.

If our hypothesis is correct and growth factors are released following microfracture of bone particles, it could be suggested that 'large' bone morsels should be used when under- 
taking this technique. Using larger particles will naturally create more microfracture when the bone is impacted. This assumes that it is beneficial to release growth factors in this way, an assumption that is easy to make in light of the recent clinical applications of recombinant BMPs to fracture repair. This recommendation is in line with current biomechanical knowledge of impacted bone stability. ${ }^{25}$

The final practical implication of this work is in relation to washing of the graft. Provided the graft has been washed after morcellisation, as suggested by biomechanical ${ }^{26-28}$ and bony ingrowth studies, ${ }^{29}$ washing should not be repeated following impaction, otherwise some of the released growth factors may be lost.

\section{Supplementary Material}

ë A table showing the full data set for each of the 40 cubes is available with the electronic version of this article on our website at www.jbjs.org.uk

The authors wish to thank C. Sutton of the University of Central Lancashire for his help in performing the statistical analysis.

No benefits in any form have been received or will be received from a commercial party related directly or indirectly to the subject of this article.

\section{References}

1. Slooff TJ, Huiskes R, van Horn J, Lemmens AJ. Bone grafting in total hip replacement for acetabular protrusion. Acta Orthop Scand 1984;55:593-6.

2. Schreurs BW, Arts JJ, Verdonschot $\mathbf{N}$, et al. Femoral component revision with use of impaction bone-grafting and a cemented polished stem. J Bone Joint Surg [Am] 2005;87-A:2499-507.

3. Gie GA, Linder L, Ling RS, et al. Impacted cancellous allografts and cement for revision total hip arthroplasty. J Bone Joint Surg [Br] 1993;75-B:14-21.

4. Schreurs BW, Busch VJ, Welten ML, et al. Acetabular reconstruction with impaction bone-grafting and a cemented cup in patients younger than fifty years old. J Bone Joint Surg [Am] 2004;86-A:2385-92.

5. Board TN, Rooney P, Kearney JN, Kay PR. Impaction allografting in revision total hip replacement. J Bone Joint Surg [Br] 2006;88-B:852-7.

6. Ullmark G, Obrant KJ. Histology of impacted bone-graft incorporation. J Arthroplasty 2002;17:150-7.

7. Ling RS, Timperley AJ, Linder L. Histology of cancellous impaction grafting in the femur: a case report. J Bone Joint Surg [Br] 1993;75-B:693-6.

8. Linder L. Cancellous impaction grafting in the human femur: histological and radiographic observations in 6 autopsy femurs and 8 biopsies. Acta Orthop Scand 2000;71:543-52.

9. Nelissen RG, Bauer TW, Weidenhielm LR, LeGolvan DP, Mikhail WE. Revision hip arthroplasty with the use of cement and impaction grafting: histological analysis of four cases. J Bone Joint Surg [Am] 1995;77-A:412-22.
10. Cook SD, Dalton JE, Tan EH, Whitecloud TS 3rd, Rueger DC. In vivo evaluation of recombinant human osteogenic protein (rhOP-1) implants as a bone graft substitute for spinal fusions. Spine 1994;19:1655-63.

11. Cook SD, Baffes GC, Wolfe MW, et al. The effect of recombinant human osteogenic protein-1 on healing of large segmental bone defects. J Bone Joint Surg [Am] 1994;76A:827-38.

12. Bilic R, Simic $\mathbf{P}$, Jelic $\mathbf{M}$, et al. Osteogenic protein-1 (BMP-7) accelerates healing of scaphoid non-union with proximal pole sclerosis. Int Orthop 2006;30:128-34.

13. Jeppson C, Astrand J, Tagil M, Aspenberg P. A combination of bisphosphonate and BMP additives in impacted bone allografts. Acta Orthop Scand 2003;74:483-9.

14. Tagil M, Jeppsson C, Aspenberg P. Bone graft incorporation: effects of osteogenic protein-1 and impaction. Clin Orthop 2000;371:240-5.

15. Tagil M, Jeppsson C, Wang JS, Aspenberg P. No augmentation of morselized and impacted bone graft by OP-1 in a weight-bearing model. Acta Orthop Scand 2003;74:742-8.

16. Karrholm J, Hourigan P, Timperley J, Razaznejad R. Mixing bone graft with OP-1 does not improve cup or stem fixation in revision surgery of the hip: 5-year follow-up of 10 acetabular and 11 femoral study cases and 40 control cases. Acta Orthop 2006;77:39-48.

17. Pietrzak WS, Woodell-May J, McDonald N. Assay of bone morphogenetic protein-2, -4 , and -7 in human demineralized bone matrix. J Craniofac Surg 2006;17:84-90.

18. Paralkar VM, Nandedkar AK, Pointer RH, Kleinman HK, Reddi AH. Interaction of osteogenin, a heparin binding bone morphogenetic protein, with type IV collagen. J Biol Chem 1990;265:17281-4.

19. Søballe K, Jensen TB, Mouzin O, Kidder L, Bechtold JE. Differential effect of a bone morphogenetic protein-7 (OP-1) on primary and revision loaded, stable implants with allograft. J Biomed Mater Res A 2004;71:569-76.

20. Granjeiro JM, Oliveira RC, Bustos-Valenzuela JC, Sogayer MC, Taga R. Bone morphogenetic proteins: from structure to clinical use. Braz J Med Biol Res 2005;38:1463-73.

21. Johnson EE, Urist MR, Finerman GA. Repair of segmental defects of the tibia with cancellous bone grafts augmented with human bone morphogenetic protein: a preliminary report. Clin Orthop 1988;236:249-57.

22. Johnson EE, Urist MR, Finerman GA. Bone morphogenetic protein augmentation grafting of resistant femoral nonunions: a preliminary report. Clin Orthop 1988;230:257-65.

23. Wang EA, Rosen V, Cordes $\mathbf{P}$, et al. Purification and characterization of other distinct bone-inducing factors. Proc Natl Acad Sci USA 1988;85:9484-8.

24. Bae HW, Zhao L, Kanim LE, et al. Intervariability and intravariability of bone morphogenetic proteins in commercially available demineralized bone matrix products. Spine 2006;31:1299-306.

25. Bolder SB, Schreurs BW, Verdonschot N, et al. Particle size of bone graft and method of impaction affect initial stability of cemented cups: human cadaveric and synthetic pelvic specimen studies. Acta Orthop Scand 2003;74:652-7.

26. Ullmark G. Bigger size and defatting of bone chips will increase cup stability. Arch Orthop Trauma Surg 2000;120:445-7.

27. Hostner J, Hultmark P, Karrholm J, Malchau H, Tveit M. Impaction technique and graft treatment in revisions of the femoral component: laboratory studies and clinical validation. J Arthroplasty 2001;16:76-82.

28. Dunlop DG, Brewster NT, Madabhushi SP, et al. Techniques to improve the shear strength of impacted bone graft: the effect of particle size and washing of the graft. J Bone Joint Surg [Am] 2003;85-A:639-46.

29. van der Donk $\mathbf{S}$, Weernink T, Buma $\mathbf{P}$, et al. Rinsing morselized allografts improves bone and tissue ingrowth. Clin Orthop 2003;408:302-10. 